\title{
PS-Modules over Ore Extensions and Skew Generalized Power Series Rings
}

\author{
Refaat M. Salem, ${ }^{1}$ Mohamed A. Farahat, ${ }^{1,2}$ and Hanan Abd-Elmalk ${ }^{3}$ \\ ${ }^{1}$ Mathematics Department, Faculty of Science, Al-Azhar University, P.O. Box 11884, Nasr City, Cairo, Egypt \\ ${ }^{2}$ Department of Mathematics and Statistics, Faculty of Science, Taif University, P.O. Box 888, Al-Hawiyah, Taif 21974, Saudi Arabia \\ ${ }^{3}$ Department of Mathematics, Faculty of Science, Ain Shams University, P.O. Box 11566, Abbasaya, Cairo, Egypt
}

Correspondence should be addressed to Mohamed A. Farahat; refaat_salem@cic-cairo.com

Received 7 December 2014; Revised 9 March 2015; Accepted 11 March 2015

Academic Editor: Ram N. Mohapatra

Copyright (c) 2015 Refaat M. Salem et al. This is an open access article distributed under the Creative Commons Attribution License, which permits unrestricted use, distribution, and reproduction in any medium, provided the original work is properly cited.

\begin{abstract}
A right $R$-module $M_{R}$ is called a PS-module if its socle, $\operatorname{Soc}\left(M_{R}\right)$, is projective. We investigate PS-modules over Ore extension and skew generalized power series extension. Let $R$ be an associative ring with identity, $M_{R}$ a unitary right $R$-module, $O=R[x ; \alpha, \delta]$ Ore extension, $M[x]_{O}$ a right $O$-module, $(S, \leq)$ a strictly ordered additive monoid, $\omega: S \rightarrow \operatorname{End}(R)$ a monoid homomorphism, $A=\left[\left[R^{S, \leq}, \omega\right]\right]$ the skew generalized power series ring, and $B_{A}=\left[\left[M^{S, \leq}\right]\right]_{\left[\left[R^{S, \leq, \omega]]}\right.\right.}$ the skew generalized power series module. Then, under some certain conditions, we prove the following: (1) If $M_{R}$ is a right PS-module, then $M[x]_{O}$ is a right PS-module. (2) If $M_{R}$ is a right PS-module, then $B_{A}$ is a right PS-module.
\end{abstract}

This paper is dedicated to Professor M. H. Fahmy on the occasion of his 68th birthday

\section{Introduction}

Throughout this paper $R$ denotes an associative ring with identity and $M_{R}$ a unitary right $R$-module. According to Nicholson and Watters [1], $M_{R}$ is called a PS-module if every simple submodule is projective and equivalently if its socle, $\operatorname{Soc}\left(M_{R}\right)$, is projective. Examples of PS-modules include nonsingular modules, regular modules in the sense of Zelmanowitz [2], and modules with zero socle. The class of PS-modules is closed under direct sums and submodules. In [3], Weimin proved that PS-modules are preserved by Morita equivalences and excellent extensions.

For any subset $X$ of $R$, denote

$$
1_{M}(X)=\{m \in M \mid m X=0\} .
$$

Theorem 1 (see [3]). The following statements are equivalent for a right $R$-module $M_{R}$ :

(1) $M_{R}$ is a PS-module.

(2) If $L$ is a maximal right ideal of $R$, then either $l_{M}(L)=0$ or $L=e R$, where $e^{2}=e \in R$.
A left PS-module ${ }_{R} M$ is defined analogously. A ring $R$ is said to be a left $P S$-ring if ${ }_{R} R$ is a PS-module. Every semiprime ring is a PS-ring. Every PP-ring is a PS-ring (where a ring $R$ is called PP-ring if every principal left ideal is projective). In particular every Baer ring is a PS-ring (where a ring $R$ is called Baer if every left (or right) annihilator is generated by an idempotent). A ring for which every simple singular module is injective is a PS-ring. If $l_{R}(J(R))=0$, then $R$ is a PS-ring. In fact $J(R) \subset L$ for every maximal right ideal so $1_{R}(L)=0$.

The notion of PS-rings is not left-right symmetric (cf. [1]). A ring $R$ is $d u o$ if each one-sided ideal of $R$ is a two-sided ideal. As a generalization of left duo rings, a ring $R$ is called weakly left duo if for every $r \in R$ there is a natural number $n(r)$ such that $R r^{n(r)}$ is a two-sided ideal of $R$. A ring $R$ is weakly duo if it is weakly right and left duo. In [3], Weimin proved that a duo ring $R$ is a PS-ring if and only if it is a right PS-ring. In [4], Dingguo generalized this result to weakly duo rings as follows: a weakly duo reduced ring $R$ is a PS-ring if and only if $R$ is a right PS-ring. 
If $R$ is a PS-ring so also are $R[x]$ and $R[[x]]$. The converse of this result is false by the following example.

Example 2 (see [1], Example 3.2). If $R=\mathbb{Z}_{4}$, then $R[x]$ and $R[[x]]$ are PS-rings but $R$ is not PS-ring.

The motivation of this paper is to investigate the PS property of Ore extension modules and the skew generalized power series extension modules. These results generalize the corresponding results for polynomial rings, generalized power series rings, and modules $[5,6]$.

\section{PS-Modules over Ore Extension Rings}

This section is devoted to study the relationship between the PS property of a right $R$-module $M_{R}$ and the PS property of the right Ore extension module $M[x]_{O}\left(M[x]_{R[x ; \alpha, \delta]}\right)$.

Let $\alpha$ be an endomorphism of $R$ and $\delta: R \rightarrow R$ an $\alpha$ derivation of $R$, that is, an additive map such that

$$
\delta(a b)=\delta(a) b+\alpha(a) \delta(b), \quad \forall a, b \in R
$$

In case $\alpha$ is the identity map, $\delta$ is called just a derivation of $R$.

The Ore extension $O=R[x ; \alpha, \delta]$ is the set of all polynomials $\sum_{i=0}^{n} a_{i} x^{i}$ with the usual sum and the following multiplication rule:

$$
x a=\alpha(a) x+\delta(a)
$$

We assume that 1 is the identity element of $O=R[x ; \alpha, \delta]$. This means that $\alpha(1)=1$ and $\delta(1)=0$. This definition of noncommutative polynomial rings with identity was first introduced by Ore [7]. Ever since the appearance of Ore's fundamental paper [7], Ore extensions have played an important role in noncommutative ring theory and many noncommutative ring theorists have investigated Ore extensions from different points of view such as ideal theory, order theory, Galois theory, and homological algebras.

For integers $i, j$ with $j \geq i \geq 0, f_{i}^{j} \in \operatorname{End}(R,+)$ will denote the map which is the sum of all possible words in $\alpha, \delta$ built with $i$ letters of $\alpha$ and $j-i$ letters of $\delta$. For instance,

$$
\begin{aligned}
f_{0}^{0} & =\operatorname{Id}_{R}, \\
f_{j}^{j} & =\alpha^{j}, \\
f_{0}^{j} & =\delta^{j}, \\
f_{j-1}^{j} & =\alpha^{j-1} \delta+\alpha^{j-2} \delta \alpha+\cdots+\delta \alpha^{j-1} .
\end{aligned}
$$

For any positive integer $n$ and $r \in R$, we have

$$
x^{n} r=\sum_{i=0}^{n} f_{i}^{n}(r) x^{i}
$$

(see [8], Lemma 4.1). This formula uniquely determines a general product of (left) polynomials in $O$ and will be used freely in what follows.
Given a right $R$-module $M_{R}, M[x]_{O}$ is a right $O$-module with the natural action of $O$ on $M[x]$ applying the above twist whenever necessary. The verification that this defines a valid $O$-module structure on $M[x]$ is almost identical to the verification that $O=R[x ; \alpha, \delta]$ is a ring and it is straightforward (see [9]).

Definition 3 (see [9]). Given a module $M_{R}$, an endomorphism $\alpha: R \rightarrow R$ and an $\alpha$-derivation $\delta: R \rightarrow R$. One says that $M_{R}$ is $\alpha$-compatible if for each $m \in M_{R}, r \in R$, one has $m r=0 \Leftrightarrow m \alpha(r)=0$. Moreover, One says that $M_{R}$ is $\delta$-compatible if for each $m \in M_{R}, r \in R$, one has $m r=0 \Rightarrow m \delta(r)=0$. If $M_{R}$ is both $\alpha$-compatible and $\delta$ compatible, one says that $M_{R}$ is $(\alpha, \delta)$-compatible.

Note that if $M_{R}$ is $\alpha$-compatible (resp., $\delta$-compatible), then $M_{R}$ is $\alpha^{i}$-compatible (resp., $\delta^{i}$-compatible) for all $i \geq 1$. It is clear that $M_{R}$ is $\alpha$-compatible (resp., $\delta$-compatible), then so is any submodule of $M_{R}$. A ring $R$ is $(\alpha, \delta)$-compatible if and only if $R_{R}$ is an $(\alpha, \delta)$-compatible module.

As an immediate consequence of Definition 3, we obtain the following.

Lemma 4. Let $M_{R}$ be an $(\alpha, \delta)$-compatible module. For each $m \in M$ and $a \in R$, one has the following:

(1) $m a=0$ if and only if $m \alpha^{n}(a)=0$ for any positive integer $n$.

(2) If $m a=0$, then $m f_{i}^{j}(a)=0$ for all $j \geq i \geq 0$.

Lemma 5 (see [10], Lemma 2.5). Let $M_{R}$ be an $(\alpha, \delta)$ compatible module, $m(x)=m_{0}+\cdots+m_{k} x^{k} \in M[x]$, and $r \in R$. If $m(x) r=0$, then $m_{i} r=0$ for each $i$.

Definition 6 (see [10]). Given a module $M_{R}$, an endomorphism $\alpha: R \rightarrow R$ and an $\alpha$-derivation $\delta: R \rightarrow R$. One says that $M_{R}$ is $(\alpha, \delta)$-Armendariz if whenever $m(x)=$ $\sum_{i=0}^{k} m_{i} x^{i} \in M[x]$ and $f(x)=\sum_{j=0}^{n} a_{j} x^{j} \in R[x ; \alpha, \delta]$ satisfy $m(x) f(x)=0$, one has $m_{i} x^{i} a_{j} x^{j}=0$ for all $i, j$.

A ring $R$ is called $(\alpha, \delta)$-Armendariz if $R_{R}$ is an $(\alpha, \delta)$ Armendariz module.

Using Lemma 5 it is easy to deduce that if $M_{R}$ is $(\alpha, \delta)$ compatible and $(\alpha, \delta)$-Armendariz, then for any $m(x)=$ $\sum_{i=0}^{k} m_{i} x^{i} \in M[x]$ and $f(x)=\sum_{j=0}^{n} a_{j} x^{j} \in R[x ; \alpha, \delta]$, $m(x) f(x)=0$ if and only if $m_{i} a_{j}=0$ for all $i, j$.

Theorem 7. Let $M_{R}$ be an $(\alpha, \delta)$-compatible and $(\alpha, \delta)$ Armendariz module. If $M_{R}$ is a PS-module, then $M[x]_{O}$ is a PS-module.

Proof. Let $L$ be a maximal right ideal of $O$. We will show that either $1_{M[x]}(L)=0$ or $L=h O$, where $h^{2}=h \in O$. Let $I$ be the set of all coefficients of all polynomials in $L$ and let $J$ be the right ideal of $R$ generated by $I$. If $J=R$, then there exist $a_{1}, \ldots, a_{n} \in I$ and $r_{1}, \ldots, r_{n} \in R$ such that

$$
1=a_{1} r_{1}+\cdots+a_{n} r_{n}
$$


Suppose that $\varphi(x)=\sum_{i=0}^{k} m_{i} x^{i} \in 1_{M[x]}(L)$ and $\varphi \neq 0$, then for every $g(x)=\sum_{j=0}^{n} a_{j} x^{j} \in L$, we have

$$
\varphi(x) g(x)=\left(\sum_{i=0}^{k} m_{i} x^{i}\right)\left(\sum_{j=0}^{n} a_{j} x^{j}\right)=0 .
$$

Since $M_{R}$ is $(\alpha, \delta)$-compatible and $(\alpha, \delta)$-Armendariz, it follows that

$$
m_{i} a_{j}=0, \quad \forall 0 \leq i \leq k, 0 \leq j \leq n .
$$

Consequently, for every $a \in I, m_{i} a=0,0 \leq i \leq k$. Hence we get

$$
\begin{aligned}
m_{i} & =m_{i} 1=m_{i}\left(a_{1} r_{1}+\cdots+a_{n} r_{n}\right) \\
& =\left(m_{i} a_{1}\right) r_{1}+\cdots+\left(m_{i} a_{n}\right) r_{n}=0,
\end{aligned}
$$

a contradiction. Then $1_{M[x]}(L)=0$. Suppose that $J \neq R$. We will show that $J$ is a maximal right ideal of $R$. Let $r \in R-J$. If $r \in L$, then $r \in I$ and so $r \in J$, a contradiction. Thus $r \notin L$. Since $L$ is a maximal right ideal of $O$,

$$
O=L+r O
$$

It follows that there exist $g(x)=\sum_{i=0}^{n} a_{i} x^{i} \in L$ and $h(x)=$ $\sum_{j=0}^{m} b_{j} x^{j} \in O$ such that $1=a_{0}+r b_{0}$. If $a_{0}=0$, then $1=r b_{0} \in$ $r R$ and so $R=J+r R$. If $a_{0} \neq 0$, then $a_{0} \in I \subseteq J$ which implies that $R=J+r R$. Hence $J$ is a maximal right ideal of $R$. Since $M_{R}$ is a PS-module, it follows that either $l_{M}(J)=0$ or $J=e R$, where $e^{2}=e \in R$. According to that we have the following two cases.

Case 1. Suppose that $\mathrm{l}_{M}(J)=0$. We will show that $\mathrm{l}_{M[x]}(L)=$ 0 . Let $\varphi(x)=\sum_{i=0}^{k} m_{i} x^{i} \in 1_{M[x]}(L)$ and $\varphi \neq 0$; then for every $g(x)=\sum_{j=0}^{n} a_{j} x^{j} \in L$, we have

$$
\varphi(x) g(x)=\left(\sum_{i=0}^{k} m_{i} x^{i}\right)\left(\sum_{j=0}^{n} a_{j} x^{j}\right)=0 .
$$

Since $M_{R}$ is $(\alpha, \delta)$-compatible and $(\alpha, \delta)$-Armendariz, it follows that $m_{i} a_{j}=0$ for all $0 \leq i \leq k$ and $0 \leq j \leq n$. Consequently, for every $a \in I, m_{i} a=0,0 \leq i \leq k$. For any $r \in J$, there exist $a_{1}, \ldots, a_{n} \in I$ and $r_{1}, \ldots, r_{n} \in R$ such that $r=a_{1} r_{1}+\cdots+a_{n} r_{n}$. Hence

$$
\begin{aligned}
m_{i} r & =m_{i}\left(a_{1} r_{1}+\cdots+a_{n} r_{n}\right) \\
& =\left(m_{i} a_{1}\right) r_{1}+\cdots+\left(m_{i} a_{n}\right) r_{n}=0,
\end{aligned}
$$

which implies that $m_{i} \in 1_{M}(J)=0$. Thus $\varphi(x)=0$, a contradiction. Hence $\mathrm{l}_{M[x]}(L)=0$.

Case 2. Suppose that $J=e R$, where $e^{2}=e \in R$. We will show that $L=e O$, where $e^{2}=e \in O$. To show that $e O \subseteq L$, we need to prove that $e \in L$. If $e \notin L$, then $O=L+e O$. Thus there exist $g(x)=\sum_{i=0}^{n} a_{i} x^{i} \in L$ and $h(x)=\sum_{j=0}^{m} b_{j} x^{j} \in O$ such that $1=a_{0}+e b_{0}$. If $a_{0}=0$, then $1=e b_{0} \in e R=J$, a contradiction. If $a_{0} \neq 0$, then $a_{0} \in I \subseteq J$ which implies that $1=a_{0}+e b_{0} \in J+e R=J$, a contradiction. Therefore $e \in L$ which implies that $e O \subseteq L$. Now we show that $L \subseteq e O$. Suppose that $g(x)=\sum_{i=0}^{n} a_{i} x^{i} \in L$; then, for all $0 \leq i \leq n$, $a_{i} \in I \subseteq J=e R$ and so $a_{i}=e a_{i}$. We have

$$
e g(x)=e \sum_{i=0}^{n} a_{i} x^{i}=\sum_{i=0}^{n}\left(e a_{i}\right) x^{i}=\sum_{i=0}^{n} a_{i} x^{i}=g(x) ;
$$

it follows that $g(x) \in e O$. Thus $L \subseteq e O$.

If $M_{R}=R_{R}$, we get the following.

Corollary 8. Let $R$ be an $(\alpha, \delta)$-compatible and $(\alpha, \delta)$ Armendariz ring. If $R$ is a right PS-ring, then $R[x ; \alpha, \delta]$ is a right PS-ring.

\section{PS-Modules over Skew Generalized Power Series Rings}

Let $(S, \leq)$ be an ordered commutative monoid. Unless stated otherwise, the operation of $S$ will be denoted additively, and the identity by 0 . Recall that $(S, \leq)$ is artinian if every strictly decreasing sequence of elements of $S$ is finite and that $(S, \leq)$ is narrow if every subset of pairwise order-incomparable elements of $S$ is finite. The following construction is due to Zhongkui [11].

Let $(S, \leq)$ be a strictly ordered monoid (i.e., if $s, s^{\prime}, t \in S$ and $s<s^{\prime}$, then $\left.s+t<s^{\prime}+t\right), R$ a ring, and $\omega: S \rightarrow \operatorname{End}(R)$ a monoid homomorphism. Consider the set $A=\left[\left[R^{S, \leq}, \omega\right]\right]$ of all maps $f: S \rightarrow R$ whose support $(\operatorname{supp}(f)=\{s \in S \mid$ $f(s) \neq 0\})$ is artinian and narrow.

For every $s \in S$ and $f, g \in A$, let

$$
\begin{aligned}
& X_{s}(f, g) \\
& \quad=\{(u, v) \in S \times S \mid u+v=s ; f(u) \neq 0, g(v) \neq 0\} .
\end{aligned}
$$

It follows from $([12], 4.1)$ that $X_{s}(f, g)$ is a finite set.

This fact allows defining the operation of multiplication (convolution) as follows:

$$
(f g)(s)=\sum_{(u, v) \in X_{s}(f, g)} f(u) \omega_{u}(g(v)),
$$

and $(f g)(s)=0$ if $X_{s}(f, g)=\phi$. With this operation and pointwise addition $A=\left[\left[R^{S, \leq}, \omega\right]\right]$ becomes a ring, which is called the ring of skew generalized power series with coefficients in $R$ and exponents in $S$.

In [13], Zhao and Jiao generalized this construction to obtain the skew generalized power series modules over skew generalized power series rings as follows.

Let $M_{R}$ be a right $R$-module; let $B$ be the set of all maps $\varphi: S \rightarrow M$ such that $\operatorname{supp}(\varphi)=\{s \in S \mid \varphi(s) \neq 0\}$ is artinian and narrow. With pointwise addition, $B=\left[\left[M^{S, \leq}\right]\right]$ is an abelian additive group. For each $f \in A=\left[\left[R^{S, \leq}, \omega\right]\right]$ and $\varphi \in B$, the set

$$
\begin{aligned}
& X_{s}(\varphi, f) \\
& \quad=\{(u, v) \in S \times S \mid u+v=s ; \varphi(u) \neq 0, f(v) \neq 0\}
\end{aligned}
$$


is finite (see [14], Lemma 1). This allows defining the scalar multiplication of the elements of $B$ by scalars from $A$ as follows:

$$
(\varphi f)(s)=\sum_{(u, v) \in X_{s}(\varphi, f)} \varphi(u) \omega_{u}(f(v))
$$

and $(\varphi f)(s)=0$ if $X_{s}(\varphi, f)=\phi$. With this operation and pointwise addition, one can easily show that $B$ is a right $A$ module, which is called the module of skew generalized power series with coefficients in $M$ and exponents in $S$.

For every $s \in S$ if we set $\omega(s)=\operatorname{Id}_{R} \in \operatorname{Aut}(R) \subset \operatorname{End}(R)$, the identity map of $R$, then $A=\left[\left[R^{S, \leq}, \omega\right]\right]=\left[\left[R^{S, \leq}\right]\right]$ is the ring of generalized power series in the sense of Ribenboim [12] and $B=\left[\left[M^{S, \leq}\right]\right]$ is the untwisted module of generalized power series in the sense of [15].

For any $r \in R$ we associated the map $c_{r} \in A$ defined by

$$
c_{r}(x)= \begin{cases}r, & \text { if } x=0, \\ 0, & \text { if } x \neq 0 .\end{cases}
$$

For any $m \in M$ and $s \in S$, we define a map $d_{m}^{s} \in B$ by

$$
d_{m}^{s}(x)= \begin{cases}m, & \text { if } x=s \\ 0, & \text { if } x \neq s\end{cases}
$$

Definition 9 (see [13]). A right $R$-module $M_{R}$ is called $\omega$ compatible whenever $m a=0$ if and only if $m \omega_{s}(a)=0$ for any $s \in S, m \in M$, and $a \in R$.

Clearly, $R$ is an $\omega$-compatible ring if and only if $R_{R}$ is an $\omega$-compatible $R$-module.

Theorem 10. Let $(S, \leq)$ be a strictly totally ordered monoid which satisfies the condition $0 \leq s$ for every $s \in S$ and let $M_{R}$ be an $\omega$-compatible module. If $M_{R}$ is a PS-module, then $B_{A}$ is a PS-module.

Proof. Let $L$ be a maximal right ideal of $A$. We will show that either $\mathrm{l}_{B}(L)=0$ or $L=h A$, where $h^{2}=h \in A$. Since $(S, \leq)$ is a strictly totally ordered monoid, $\operatorname{supp}(f)$ is a nonempty wellordered subset of $S$, for every $0 \neq f \in A$. We denote by $\pi(f)$ the smallest element of support $f$.

For any $s \in S$, set

$$
\begin{aligned}
I_{s} & =\{f(s) \mid f \in L, \pi(f)=s\} \subset R, \\
I & =\bigcup_{s \in S} I_{s} .
\end{aligned}
$$

Let $J$ be the right ideal of $R$ generated by $I$. If $J=R$, then there exist $s_{1}, \ldots, s_{n} \in S, f_{1}, \ldots, f_{n} \in L$, and $r_{1}, \ldots, r_{n} \in R$ such that

$$
1=f_{1}\left(s_{1}\right) r_{1}+\cdots+f_{n}\left(s_{n}\right) r_{n}
$$

where $f_{i}\left(s_{i}\right) \in I_{s_{i}}$ and $\pi\left(f_{i}\right)=s_{i}$, for every $1 \leq i \leq n$. We will show that $\mathrm{l}_{B}(L)=0$. Suppose that $\varphi \in 1_{B}(L)$ and $\varphi \neq 0$. Then $\operatorname{supp}(\varphi)$ is a nonempty well-ordered subset of $S$. Let $t=\pi(\varphi)$; if

$$
\varphi(t) \omega_{t}\left(f_{i}\left(s_{i}\right)\right) \neq 0, \quad \text { for some } 1 \leq i \leq n,
$$

then

$$
\begin{aligned}
\left(\varphi f_{i}\right)\left(t+s_{i}\right) & =\sum_{(u, v) \in X_{t+s_{i}}\left(\varphi, f_{i}\right)} \varphi(u) \omega_{u}\left(f_{i}(v)\right) \\
& =\varphi(t) \omega_{t}\left(f_{i}\left(s_{i}\right)\right) \neq 0 .
\end{aligned}
$$

This means that $\varphi f_{i} \neq 0$ for some $1 \leq i \leq n$, a contradiction. Thus

$$
\varphi(t) \omega_{t}\left(f_{i}\left(s_{i}\right)\right)=0, \quad \forall 1 \leq i \leq n
$$

Since $M_{R}$ is an $\omega$-compatible module, we get

$$
\varphi(t) f_{i}\left(s_{i}\right)=0, \quad \forall 1 \leq i \leq n .
$$

Consequently

$$
\begin{aligned}
\varphi(t) & =\varphi(t) 1=\varphi(t)\left(f_{1}\left(s_{1}\right) r_{1}+\cdots+f_{n}\left(s_{n}\right) r_{n}\right) \\
& =\left(\varphi(t) f_{1}\left(s_{1}\right)\right) r_{1}+\cdots+\left(\varphi(t) f_{n}\left(s_{n}\right)\right) r_{n}=0,
\end{aligned}
$$

a contradiction. Thus $1_{B}(L)=0$. Suppose that $J \neq R$. We will show that $J$ is a maximal right ideal of $R$. Let $r \in R-J$. If $c_{r} \in L$, then $r=c_{r}(0) \in I_{0} \subset I$ and so $r \in J$, a contradiction. Therefore $c_{r} \notin L$. Since $L$ is a maximal right ideal of $A$,

$$
A=L+c_{r} A
$$

It follows that there exist $f \in L$ and $g \in A$ such that $c_{1}=$ $f+c_{r} g$. Thus

$$
\begin{aligned}
1 & =c_{1}(0)=f(0)+\left(c_{r} g\right)(0)=f(0)+r \omega_{0}(g(0)) \\
& =f(0)+r g(0) .
\end{aligned}
$$

If $f(0)=0$, then $1=r g(0) \in r R$. So, $R=J+r R$.

If $f(0) \neq 0$, then $0 \in \operatorname{supp}(f)$. Since $0 \leq s$ for every $s \in S, \pi(f)=0$. Thus $f(0) \in I_{0} \subset I \subset J$, which implies that $R=J+r R$.

Hence $J$ is a maximal right ideal of $R$. Since $M_{R}$ is a PSmodule, it follows that either $l_{M}(J)=0$ or $J=e R$, where $e^{2}=e \in R$. According to that we have the following two cases.

Case 1. Suppose that $\mathrm{l}_{M}(J)=0$. We will show that $\mathrm{l}_{B}(L)=0$. Let $\varphi \in 1_{B}(L)$ and $\varphi \neq 0$. Then $\operatorname{supp}(\varphi)$ is a nonempty wellordered subset of $S$. Let $s=\pi(\varphi)$. For any $r \in J$, there exist $s_{1}, \ldots, s_{n} \in S, f_{1}, \ldots, f_{n} \in L$, and $r_{1}, \ldots, r_{n} \in R$ such that

$$
r=f_{1}\left(s_{1}\right) r_{1}+\cdots+f_{n}\left(s_{n}\right) r_{n},
$$

where $f_{i}\left(s_{i}\right) \in I_{s_{i}}$ and $\pi\left(f_{i}\right)=s_{i}$, for every $1 \leq i \leq n$. Since $\varphi \in 1_{B}(L), f_{1}, \ldots, f_{n} \in L$, we get $\varphi f_{i}=0$ for every $1 \leq i \leq n$. If

$$
\varphi(s) \omega_{s}\left(f_{i}\left(s_{i}\right)\right) \neq 0, \quad \text { for some } 1 \leq i \leq n,
$$


then

$$
\begin{aligned}
\left(\varphi f_{i}\right)\left(s+s_{i}\right) & =\sum_{(u, v) \in X_{s+s_{i}}\left(\varphi, f_{i}\right)} \varphi(u) \omega_{u}\left(f_{i}(v)\right) \\
& =\varphi(s) \omega_{s}\left(f_{i}\left(s_{i}\right)\right) \neq 0 .
\end{aligned}
$$

This means that $\varphi f_{i} \neq 0$ for some $1 \leq i \leq n$, a contradiction. Thus

$$
\varphi(s) \omega_{s}\left(f_{i}\left(s_{i}\right)\right)=0, \quad \forall 1 \leq i \leq n .
$$

Since $M_{R}$ is an $\omega$-compatible module, we get

$$
\varphi(s) f_{i}\left(s_{i}\right)=0, \quad \forall 1 \leq i \leq n .
$$

Consequently

$$
\begin{aligned}
\varphi(s) r & =\varphi(s)\left(f_{1}\left(s_{1}\right) r_{1}+\cdots+f_{n}\left(s_{n}\right) r_{n}\right) \\
& =\left(\varphi(s) f_{1}\left(s_{1}\right)\right) r_{1}+\cdots+\left(\varphi(s) f_{n}\left(s_{n}\right)\right) r_{n} \\
& =0 .
\end{aligned}
$$

Therefore, $\varphi(s) \in 1_{M}(J)=0$ and $\pi(\varphi)=s$. Thus $\varphi=0$, a contradiction. Hence $\mathrm{l}_{B}(L)=0$.

Case 2. Suppose that $J=e R$, where $e^{2}=e \in R$. We will show that $L=c_{e} A$, where $\left(c_{e}\right)^{2}=c_{e} \in A$. To show that $c_{e} A \subseteq L$, we need to prove that $c_{e} \in L$. If $c_{e} \notin L$, then $A=L+c_{e} A$. Thus there exist $f \in L$ and $g \in A$ such that $c_{1}=f+c_{e} g$. Thus

$$
\begin{aligned}
1 & =c_{1}(0)=f(0)+\left(c_{e} g\right)(0)=f(0)+e \omega_{0}(g(0)) \\
& =f(0)+e g(0) .
\end{aligned}
$$

If $f(0)=0$, then $1=e g(0) \in e R=J$, a contradiction.

If $f(0) \neq 0$, then $0 \in \operatorname{supp}(f)$. Since $0 \leq s$ for every $s \in S, \pi(f)=0$. Thus $f(0) \in I_{0} \subset I \subset J$, which implies that $f(0) \in J$ and $J=e R$. Hence $1=f(0)+e g(0) \in J+e R=J$, a contradiction. Therefore $c_{e} \in L$ which implies that $c_{e} A \subseteq L$.

Conversely, suppose that $f \in L$ and $\pi(f)=s$; then $f(s) \in$ $I_{s} \subset I \subset J=e R$ and so $f(s)=e f(s)$. We claim that $f(u)=$ $e f(u)$ for any $u \in \operatorname{supp}(f)$.

Suppose that $f(v)=e f(v)$ for each $v<u$. Consider the following element $f_{u} \in A$ defined by

$$
f_{u}(x)= \begin{cases}f(x), & x<u \\ 0, & x \geq u .\end{cases}
$$

Thus $\pi\left(f-f_{u}\right)=u$. By hypothesis it is easy to see that $f_{u}=$ $c_{e} f_{u} \in c_{e} A \subset L$. Thus $f-f_{u} \in L$. By analogy with the proof above, it follows that

$$
\left(f-f_{u}\right)(u)=e\left(f-f_{u}\right)(u),
$$

which implies that $f(u)=e f(u)$. Thus our claim holds. Therefore

$$
\begin{aligned}
\left(c_{e} f\right)(t) & =\sum_{(u, v) \in X_{t}\left(c_{e}, f\right)} c_{e}(u) \omega_{u}(f(v)) \\
& =c_{e}(0) \omega_{0}(f(t))=e f(t)=f(t) .
\end{aligned}
$$

Hence $f=c_{e} f \in c_{e} A$. Thus $L=c_{e} A$ and the result follows since $c_{e}$ is an idempotent of $A$.
As a special case of the last result if we set $M_{R}=R_{R}$ we get the following.

Corollary 11. Let $(S, \leq)$ be a strictly totally ordered monoid which satisfies the condition that $0 \leq s$ for every $s \in S$ and let $R$ be an $\omega$-compatible ring. If $R$ is a right PS-ring, then $A=\left[\left[R^{S, \leq}, \omega\right]\right]$ is a right PS-ring.

If we set $\omega(s)=\operatorname{Id}_{R}$, for every $s \in S$, we get the following as a corollary.

Corollary 12 (see [6], Theorem 1). Let $(S, \leq)$ be a strictly totally ordered monoid which satisfies the condition that $0 \leq s$ for every $s \in S$. If $M_{R}$ is a PS-module, then $\left[\left[M^{S, \leq}\right]\right]_{\left[\left[R^{S, \leq}\right]\right]}$ is a PS-module.

If $M_{R}=R_{R}$, we get the following as a corollary.

Corollary 13 (see [5], Theorem 4). Let $(S, \leq)$ be a strictly totally ordered monoid which satisfies the condition that $0 \leq s$ for every $s \in S$. If $R$ is a right PS-ring, then $\left[\left[R^{S, \leq}\right]\right]$ is a right PS-ring.

\section{Conflict of Interests}

The authors declare that there is no conflict of interests regarding the publication of this paper.

\section{References}

[1] W. K. Nicholson and J. F. Watters, "Rings with projective socle," Proceedings of the American Mathematical Society, vol. 102, no. 3, pp. 443-450, 1988.

[2] J. Zelmanowitz, "Regular modules," Transactions of the American Mathematical Society, vol. 163, pp. 341-355, 1972.

[3] X. Weimin, "Modules with projective socles," Rivista di Matematica della Università di Parma, vol. 1, no. 5, pp. 311-315, 1992.

[4] W. Dingguo, "Modules with flat socles and almost excellent extensions," Acta Mathematica Vietnamica, vol. 21, no. 2, pp. 295-301, 1996.

[5] Z. Liu and F. Li, "PS-rings of generalized power series," Communications in Algebra, vol. 26, no. 7, pp. 2283-2291, 1998.

[6] Z. Liu, "PS-modules over rings of generalized power series," Northeastern Mathematical Journal, vol. 18, no. 3, pp. 254-260, 2002.

[7] Ø. Ore, "Theory of non-commutative polynomials," The Annals of Mathematics, vol. 34, no. 3, pp. 480-508, 1933.

[8] T. Y. Lam, A. Leroy, and J. Matczuk, "Primeness, semiprimeness and prime radical of Ore extensions," Communications in Algebra, vol. 25, no. 8, pp. 2459-2506, 1997.

[9] S. Annin, "Associated primes over Ore extension rings," Journal of Algebra and its Applications, vol. 3, no. 2, pp. 193-205, 2004.

[10] E. Hashemi, "Extensions of BAEr and quasi-BAEr modules," Iranian Mathematical Society-Bulletin, vol. 37, no. 1, pp. 1-13, 2011.

[11] L. Zhongkui, "Triangular matrix representations of rings of generalized power series," Acta Mathematica Sinica (English Series), vol. 22, no. 4, pp. 989-998, 2006. 
[12] P. Ribenboim, "Semisimple rings and von Neumann regular rings of generalized power series," Journal of Algebra, vol. 198, no. 2, pp. 327-338, 1997.

[13] R. Zhao and Y. Jiao, "Principal quasi-Baerness of modules of generalized power series," Taiwanese Journal of Mathematics, vol. 15, no. 2, pp. 711-722, 2011.

[14] L. Zhongkui, "A note on Hopfian modules," Communications in Algebra, vol. 28, no. 6, pp. 3031-3040, 2000.

[15] K. Varadarajan, "Generalized power series modules," Communications in Algebra, vol. 29, no. 3, pp. 1281-1294, 2001. 


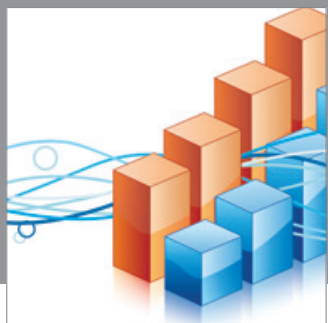

Advances in

Operations Research

mansans

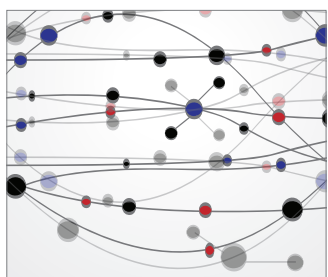

The Scientific World Journal
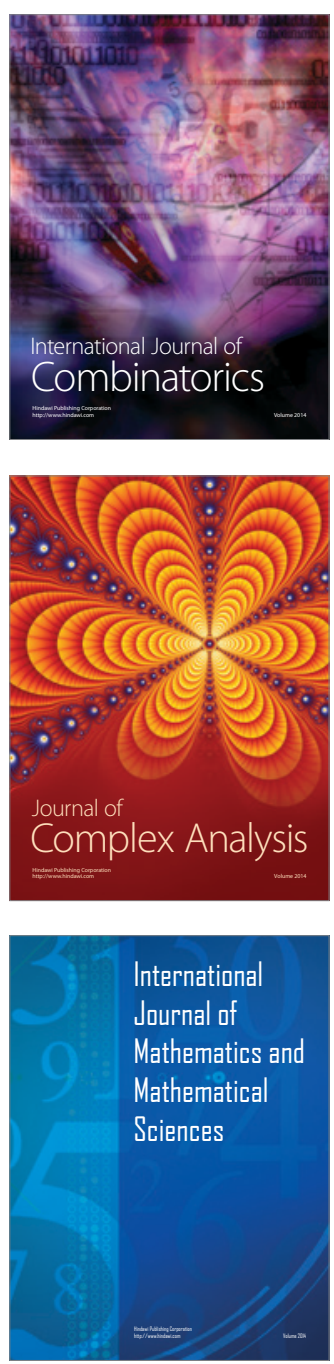
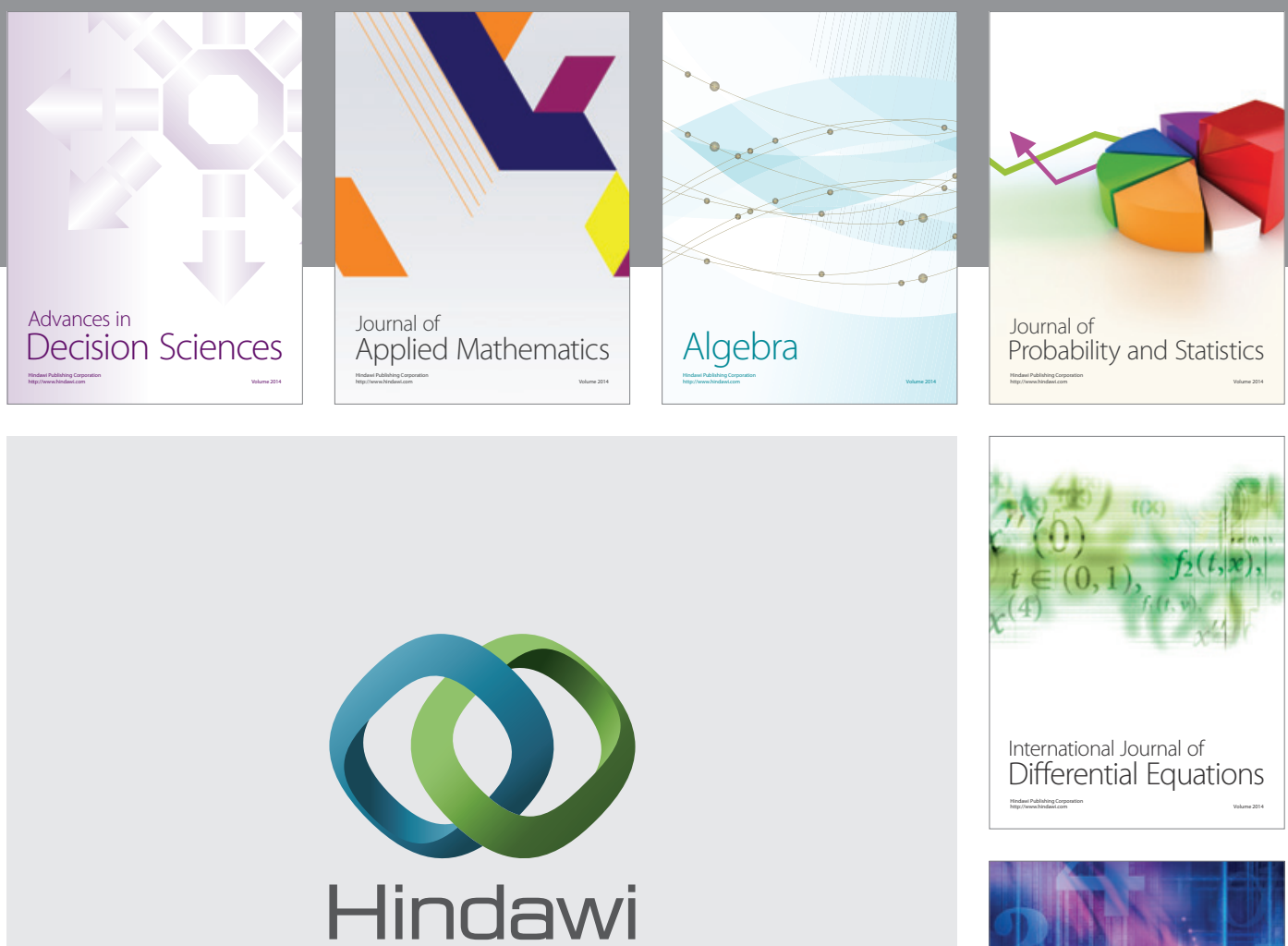

Submit your manuscripts at http://www.hindawi.com
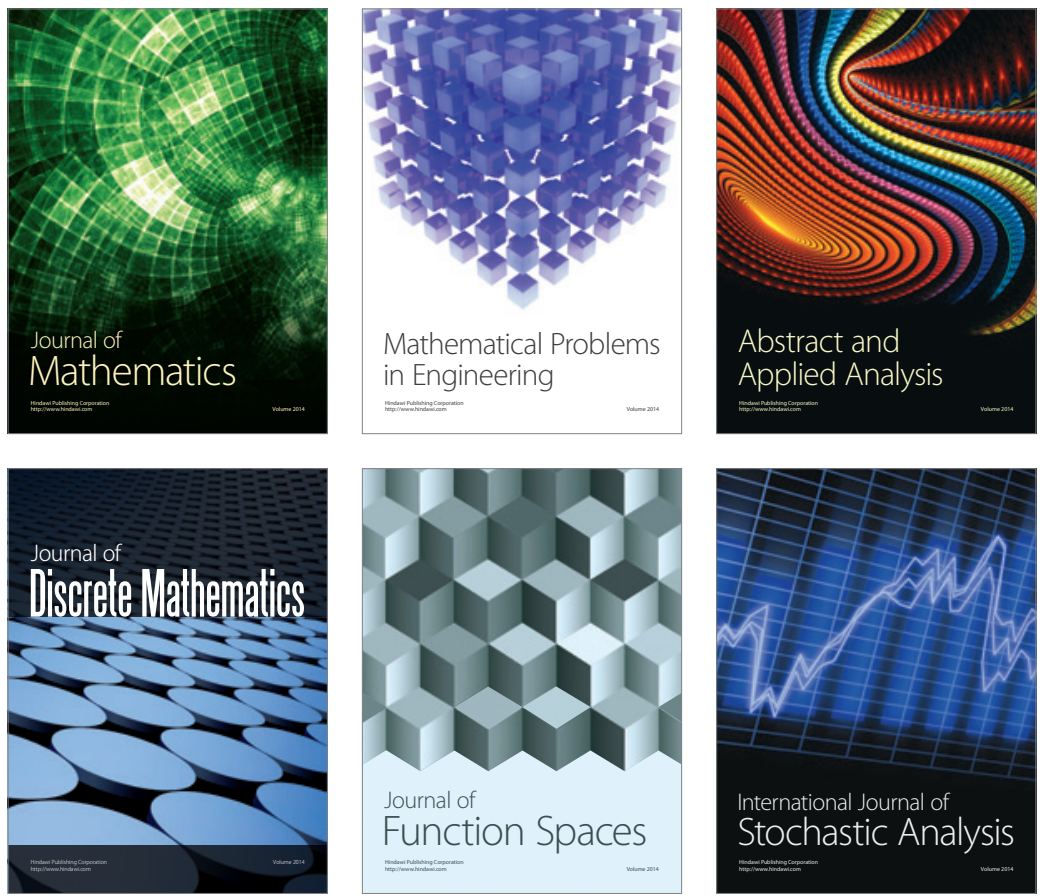

Journal of

Function Spaces

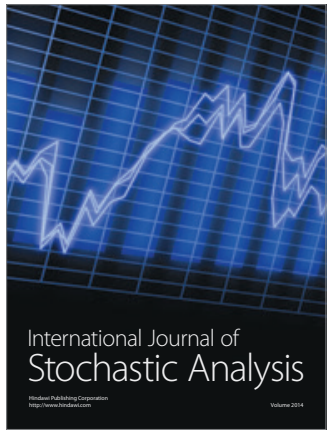

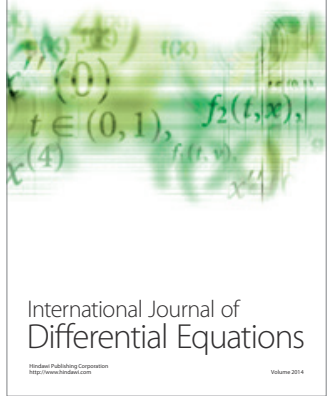
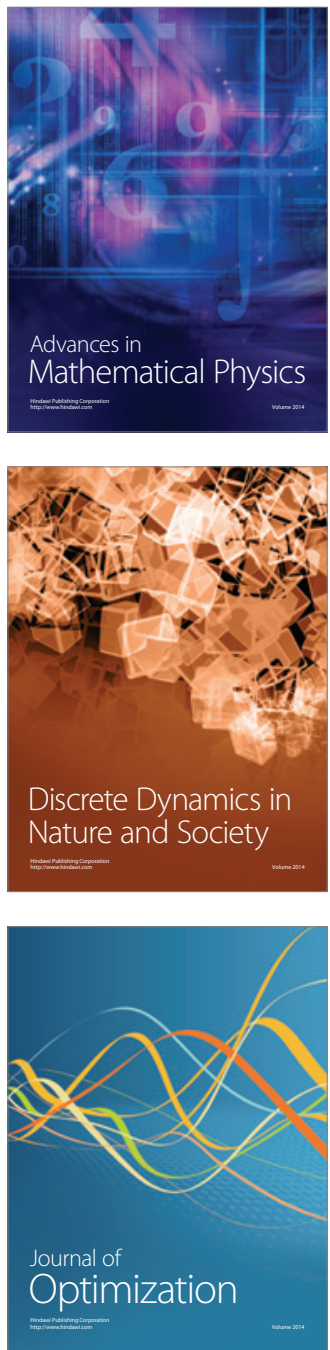